\title{
Estilos de comunicación como predictores del estrés y el afrontamiento diádico en matrimonios puertorriqueños ${ }^{1}$
}

\author{
Ruth Nina-Estrella ${ }^{2}$ y Jesús J. Marrero Centeno ${ }^{3}$ \\ Universidad de Puerto Rico (Puerto Rico)
}

Recibido: 26/06/2020Ａceptado: 19/06/2020

\begin{abstract}
Resumen
Objetivo. Evaluar la posible relación de los estilos de comunicación (positivo, negativo y reservado) con el estrés y el afrontamiento diádico en las relaciones de pareja. Método. Se empleó un estudio cuantitativo no experimental en el que participaron 121 personas casadas, seleccionadas por disponibilidad. La mayoría de los participantes estaban en su primer matrimonio, y tenían una edad promedio de 42 años. Aceptaron contestar los siguientes instrumentos: (a) inventario de estilo de comunicación marital (ECOM) y (b) escala de estrés en la relación marital. Resultados. El análisis mediante regresión múltiple evidenció que los estilos de comunicación positivo y reservado son predictores del afrontamiento diádico. Se observó también que el estilo de comunicación negativo es un predictor significativo del estrés en las relaciones de pareja. Conclusión. Se sustenta la importancia de la comunicación en las relaciones de pareja y de desarrollar estilos de comunicación positivos para afrontar las situaciones estresantes.
\end{abstract}

Palabras clave. Estilos de comunicación, estrés, afrontamiento diádico, relación de pareja.

\section{Communication Styles as Stress Predictors and Dyadic Coping in Puerto Rican Marriages}

\begin{abstract}
Objetive. To evaluate the possible relationship between the communication styles (positive, negative and reserved), stress and dyadic coping. Method. Considering this purpose, a non-experimental quantitative study was proposed in which 121 married people participated based on their availability. Most of the participants were in their first marriage, and averaged 42 years old. The participants agreed to answer the following instruments: (a) Marital Communication Style Inventory and (b) Marital Relationship Stress Scale. Results. Positive and reserved communication styles are predictors of dyadic coping. It was also observed that the negative communication style is a significant predictor of stress in relationships. Conclusion. These results support the relevance of communication in relationships and the importance of developing positive communication styles to cope with stressful situations.
\end{abstract}

Keywords. Communication styles, stress, dyadic coping, relationship.

Proyecto Comunicación y Afrontamiento Diádico.

2 Doctora en Psicología Social, Universidad de Puerto Rico, Departamento de Psicología. Correo de correspondencia: ruth.nina1@upr.edu

3 Doctor en Psicología Industrial Organizacional. 


\title{
Estilos de comunicação como preditores do stress e o afrontamento diádico em matrimônios porto-riquenhos
}

\begin{abstract}
Resumo
Escopo. Avaliar a possível relação dos estilos de comunicação (positivo, negativo e reversado) com o stress e o afrontamento diádico nas relações de casal. Metodologia. Foi empregado um estudo quantitativo não experimental no que participaram 121 pessoas casadas, selecionadas por disponibilidade. A maioria dos participantes estavam no seu primeiro matrimonio, e tinham uma idade média de 42 anos. Aceitaram contestar os seguintes instrumentos: (a) inventário de estilo de comunicação marital (ECOM) e (b) escada de stress na relação marital. Resultados. A análise mediante regressão múltipla evidenciou que os estilos de comunicação positivo e reservado são preditores do afrontamento diádico. Foi observado também que o estilo de comunicação negativo é um preditor significativo do stress nas relações de casal. Conclusão. Foi sustentada a importância da comunicação nas relações de casal e de desenvolver estilos de comunicação positivos para afrontar as situações estressantes.
\end{abstract}

Palavras-chave. Estilos de comunicação, stress, afrontamento diádico, relação de casal.

\section{Introducción}

La comunicación es un factor importante en las relaciones de pareja, que puede contribuir al éxito de esta. La comunicación y las relaciones se entrelazan dentro de un proceso en el cual se recrea un mundo de significados (Sillars y Vangelisti, 2018). De hecho, toda comunicación tiene un propósito, causa un resultado, crea una atmósfera, establece una identidad y se sostiene en el desarrollo de la relación en la vida cotidiana, reflejando la calidad de la relación de pareja.

La comunicación marital se centra en la relación diádica, en la cual existe un lazo afectivo que evoca una interdependencia entre dos (Bradbury y Karney, 2014), en tanto que cada integrante de la relación construye un sentido de estar en pareja, de ahí que se produzcan interacciones y experiencias que influyen en la pareja. De esta manera, la relación emerge y asume cambios y transiciones a través de su propia historia de vida (Duck y McMahan, 2010; Finkel, Simpson y Eastwink, 2017).

El acto de comunicar comprende diversos elementos, como el contenido, la intención, la valencia y el estilo de comunicación. Dado que el estilo de la comunicación es un tema poco estudiado en el campo de las relaciones de pareja, aunque se reconoce su significado al enmarcar el contenido de la comunicación (Kelley, 2012), en este artículo se evalúa y analiza el tipo de estilo de comunicación utilizado cuando la pareja enfrenta una situación estresante.

Desde la década de los ochenta se inicia el estudio del estilo de comunicación como un constructo que se refiere a la forma en que cada persona se comunica, es decir, de la comunicación interpersonal (Norton, 1978; 1983). Estas investigaciones han tenido repercusión en diversos campos: educativos, laborales y de comunicación intercultural. Entre las propiedades que se le atribuyen al concepto, se resaltan las siguientes: (a) un patrón recurrente de conductas, y (b) cada persona tiene una disposición personal de comunicarse de una manera particular (Waldherr y Muck, 2011).

Al ser una variable multidimensional, el estilo de comunicación implica que, según la situación que enfrente la pareja, ambos deben ajustarse al estilo del otro integrante para que la relación tenga continuidad. Por lo que tanto el contenido como la forma en que se comunica la pareja en la relación son elementos determinantes del bienestar psicológico, la satisfacción, la calidad de vida y la salud de sus integrantes (Sánchez-Aragón y DíazLoving, 2003; Sánchez y Watty, 2017). Por otro lado, en un estilo abierto de comunicación, en el que se expresan sentimientos o pensamientos positivos, la persona puede mostrar mayor satisfacción con la relación de pareja. En cambio, la insatisfacción puede estar asociada a estilos de comunicación negativos (Wijnberg-Williams, Van de Wiel, Kamps y Hoekstra-Weebers, 2015). 
El estilo de comunicación también se caracteriza por ser observable. Es decir, en cada interacción de la comunicación, la forma en que se exprese el contenido es un elemento que puede ser observable por otros y se reproduce en diferentes estilos en los que existe una interdependencia que varía según el contexto social en que se presente. En la relación de pareja, el estilo de comunicación determinará la realidad particular en cada uno de los miembros, quienes establecen la interacción, la afectividad y su construcción (Uribe, García y Ramírez, 2011).

La calidad de la relación marital se refleja en el estilo de comunicación, el cual contribuye incluso en el mantenimiento y la estabilidad de la díada; aunque puede conllevar malentendidos y provocar situaciones conflictivas. Si bien es un tema fundamental en las relaciones de pareja, es poco estudiado, de acuerdo con la literatura consultada referente a las poblaciones latinas.

Al hacer una revisión sobre los estilos de comunicación en las relaciones de parejas mexicanas, Sánchez-Aragóny Díaz-Loving (2003)concluyen que la mayoría utilizan estilos de comunicación positivos. Esto debido a que les permiten fortalecer los vínculos, mejorar el desarrollo personal y crecer como pareja. Mientras que los estilos de comunicación negativos provocan la disminución de la escucha. Por ende, se reduce la capacidad de atender y comprender las necesidades del otro.

Por su parte, Uribe et al. (2011) analizan la relación entre la satisfacción sexual y los estilos de comunicación. En su estudio, estos autores encontraron que existen diferencias significativas entre hombres y mujeres, lo cual determina que el uso del estilo de comunicación influye directamente en la satisfacción sexual. De manera similar, Sánchez y Watty (2017) analizan la relación entre el contenido, el estilo y la satisfacción marital. Estas autoras puntualizan que el estilo depende del contenido de lo que se habla. En este caso, las mujeres consideran que usar el estilo de comunicación negativo y reservado se asocia con conversaciones conflictivas. En cambio, los hombres manifiestan sentirse insatisfechos al hablar sobre los hijos, contenido que está relacionado con un estilo reservado y negativo. Por último, las autoras sustentan que el estilo de comunicación positivo es el que más que contribuye a la satisfacción de la relación.
En cuanto a los instrumentos de medición sobre estilos de comunicación, predominan los autorreportes. La escala de estilos de comunicación (ECOM; Nina, 1991; 1992), diseñada para medir el estilo de comunicación propio y de la pareja, tiene cuatro dimensiones en el contexto de la pareja: estilo positivo, estilo negativo, estilo reservado y estilo violento. De estos estudios se estableció que en la pareja se reproducen diversos estilos que son aprendidos a través de normas culturales y de socialización, según el contenido de la comunicación. Por otra parte, SánchezAragón y Díaz-Loving (2003) identificaron estilos de comunicación propios de la cultura mexicana, como: empático y social expresivo, social afiliativo, social automodificador, simpático y social normativo, maquiavélico, chismoso e hiriente e irritante expresivo. Esto demuestra que las formas o patrones, más que los tópicos y el contenido de la comunicación, cumplen un papel fundamental en la manera en que se relacionan las personas.

Conceptos relacionados con dichos estilos son el estrés y el afrontamiento diádico, que emergen en la década de los noventa, cuando se ampliaron los estudios sobre estos en el campo de la pareja, la familia y las comunidades (Falconier y Kuhn, 2019). Ambos conceptos forman parte del proceso interpersonal que se produce entre los integrantes de la relación. Para Bodenmann (1995; 2005), el estrés diádico se define como todo encuentro que produce estrés en la pareja, que puede afectar la relación de diversas maneras y desencadenar en alguna forma de afrontamiento. En su estudio, desde un contexto diádico, es relevante considerar diversos elementos, como el estrés en uno de los integrantes, la percepción de la pareja sobre el estrés y la reacción de uno de los integrantes sobre el estrés en su pareja. Por otra parte, el afrontamiento diádico se refiere a la serie de pensamientos y acciones que capacitan a las personas para manejar situaciones difíciles o de tensión en la relación (Bodenmann, Pihet y Kayser, 2006). También puede expresarse mediante acciones conductuales positivas o negativas (Razak, Hoesni, Zaharia y Ismail, 2015).

Desde el modelo teórico de Bodenmann (1995; 2005), los conceptos de estrés y el afrontamiento se vinculan. Dicho modelo se enfoca en el impacto del estrés cotidiano en el funcionamiento de la pareja, el cual puede ser agudo o crónico. Además, parte de la premisa de que estos constructos son parte 
de procesos psicológicos presentes en la relación y que involucran a ambos cónyuges a nivel individual y de pareja (Bodenmann, Hilpert, Nussbeck y Bradbury, 2014; Sillars y Vangelisti, 2018).

Ante el estrés, existen diversas estrategias de afrontamiento que pueden manejarse a nivel individual, pero la estrategia que se utilice dependerá de la naturaleza del estresante y de las circunstancias en las que se produce (Randall y Bodenmann, 2009). También es importante comprender que en el proceso de afrontamiento en la relación de pareja influyen diversos factores, tales como: contexto social, tipo de estresor, grado de reconocimiento de la pareja, causas del estrés, factores de personalidad, motivaciones y relacionales (Falconier y Kuhn, 2019); así como la cultura, como elemento determinante en la manera en que la pareja maneja el estrés y el afrontamiento diádico (Falconier, Randall y Bodenmann, 2016). De ahí que el estilo de comunicación, de orientación cultural individual o colectiva, puede influir en las respuestas de afrontamiento de la pareja y en la apreciación del estrés.

En el modelo sistémico transaccional de Bodenmann (1995; 2005), el proceso de estrés y afrontamiento está conformado por una serie de acciones y transacciones, las cuales afectan y modifican el sistema en el que los individuos están insertados (Cutrona, Bodenmann, Randall, Clavel y Johnson, 2018). En eventos estresantes, el uso de la comunicación sobre el estresor a la pareja, la rápida reacción de la pareja, los recursos de la pareja para su manejo y la apreciación del estresor son parte del proceso en que la díada busca afrontar su situación estresante. Además, es un proceso sobre cómo la pareja comunica el estrés, cómo responde a cada uno de los signos estresantes y cómo enfrentan las adversidades (Leuchtmann et al., 2018). Debido a ello, es importante desarrollar intervenciones enfocadas en la pareja, con el propósito de educarla sobre el manejo del estrés y a desarrollar destrezas de comunicación para poder manejar situaciones estresantes (Lavner y Bradbury, 2017).

Las investigaciones sobre el estrés, el afrontamiento diádico y la comunicación de la pareja establecen que el estrés afecta directamente a la comunicación, así como a la satisfacción y a la calidaddela relación(Kelley, 2012). Lacomunicación se ve afectada por dos tipos de estresores: (a) estrés externo (situaciones laborales, conflictos con los vecinos o problemas económicos), y (b) estrés relacional (actitudes, hábitos y necesidades que reproducen tensiones entre los integrantes). Por lo que, cuando los integrantes de la pareja evitan hablar sobre el estrés o sobre cómo afrontarlo, pueden asumir conductas hostiles, agresivas o de insatisfacción (Karney y Bradbury, 1995). En consecuencia, dependiendo del significado que le brinden al estresor, los cónyuges harán el esfuerzo de mantener la relación (Ledermann, Bodenmann, Rudaz y Bradbury, 2010).

Para Cutrona et al. (2018), una de las situaciones más estresantes que vive la pareja es hablar sobre situaciones laborales, debido al efecto desbordamiento (spillover), o traspaso de estrés, que produce una interacción negativa entre los integrantes de la relación. Al estar estresada la pareja, o uno de sus integrantes, se reproducen conductas hostiles o agresivas, particularmente si los integrantes no presentan estilos de comunicación que faciliten la apertura y la solución de conflictos. Al estar expuestos a escenarios estresantes, se afectan las habilidades de comunicación y la funcionabilidad de sus integrantes (Hilpert et al., 2016).

Recientemente, Nina (2016) realizó un estudio sobre el estrés y el afrontamiento diádico en la relación de pareja puertorriqueña. Con una muestra de 311 personas casadas, se analizaron las causas del estrés, las vivencias que provoca este evento y las estrategias de afrontamiento que asumen los cónyuges ante las situaciones estresantes. Se encontró que la situación económica es el mayor estresor. Por otra parte, se comprobó que la comunicación entre la pareja es una de las estrategias de afrontamiento más recurrente que utiliza la díada. Se observó que los cónyuges, al sentir estrés, realizan actividades distractoras, reaccionan con alguna respuesta fisiológica o se aíslan (requieren que la pareja les brinde un espacio, esto quiere decir que se aparte). Los resultados del estudio coinciden con la postura teórica del modelo propuesto por Bodenmann (2005).

El estrés y el afrontamiento diádico son elementos de la cotidianidad, de modo que las formas que asuman los integrantes de la pareja para comunicarse serán vitales para el funcionamiento de la relación. Así que los estilos de comunicación de la pareja pueden influir en el manejo del estrés y las estrategias de afrontamiento diádico. Sin 
embargo, la literatura demuestra la ausencia de estudios previos que examinen el comportamiento de estas variables en el contexto latinoamericano. Por ello, se analizó la relación entre los estilos de comunicación, el estrés y el afrontamiento diádico en matrimonios puertorriqueños (ver figura 1).

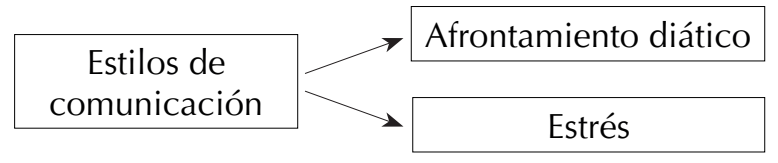

Figura 1. Modelo hipotetizado de los estilos de comunicación, el estrés y afrontamiento diádico.

Fuente: elaboración propia.

El acercamiento al estudio del estilo de la comunicación, el estrés y el afrontamiento diádico permite una mejor comprensión al tema y a su vez conocer su efecto en la calidad de la relación de pareja. Esto con el fin de identificar los mejores estilos de comunicación, que pueden contribuir a la estabilidad, la calidad y el bienestar psicológico de la relación de pareja.

\section{Método}

\section{Diseño}

El estudio se basó en una investigación cuantitativa no experimental de cohorte transversal.

\section{Participantes}

La muestra utilizada fue no probabilística y estuvo compuesta por 121 personas casadas (44 hombres y 77 mujeres). En su mayoría, los participantes eran mujeres $(63.6 \%)$. La edad fluctuó entre los 20 y los 73 años $(M=42.07, D E=12.01)$. Respecto al nivel de educación, la mayor parte de la muestra contaba con un grado universitario completado $(67.8 \%)$. Al momento en que se llevó a cabo la investigación, la mayoría de los participantes estaban empleados (82.5\%).

Como parte de los criterios de inclusión, debían cumplir con lo siguiente: (a) no estar en consejería o terapia psicológica, (b) no vivir una situación de violencia doméstica, (c) estar casado legalmente, y (d) ser mayor de edad.
Los participantes, en su mayoría estaban en su primer matrimonio $(79.3 \%)$ y tenían hijos $(77.5 \%)$. El tiempo de casados con su pareja fluctuaba entre 1 y 50 años $(M=14.63, D E=11.11)$. Por último, gran parte de la muestra practicaba alguna religión $(81 \%)$ : catolicismo $(41.7 \%)$ y protestantismo (33.3\%) (Para mayor información ver tabla 1).

Tabla 1 Características sociodemográficas de la muestra ( $n$ $=121$ )

n $\%$

\section{Género}

Masculino

$44 \quad 36.4$

Femenino

$77 \quad 63.6$

Educación

Escuela superior incompleta

$1 \quad 0.8$

Escuela superior completa

$13 \quad 10.7$

Universidad incompleta

$24 \quad 19.8$

Universidad completa

$82 \quad 67.8$

¿Cuál matrimonio es este?

Primer matrimonio

$96 \quad 79.3$

Segundo matrimonio

$23 \quad 19.0$

Tercer matrimonio

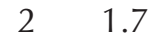

¿Tienes hijos?

Sí

$93 \quad 77.5$

No

$27 \quad 22.5$

Trabajas

Sí

$99 \quad 82.5$

No

$20 \quad 16.7$

Religión

Católica

$\begin{array}{ll}50 & 41.7\end{array}$

Protestante

$\begin{array}{ll}40 & 33.3\end{array}$

Otra

$\begin{array}{ll}8 & 6.7\end{array}$

No practica ninguna religión

$22 \quad 18.3$

Fuente: elaboración propia. 


\section{Instrumentos}

\section{Inventario de estilo de comunicación marital (ECOM).}

ECOM, en su versión original, mide 43 adjetivos que describen la manera en la que los integrantes de una pareja se comunican entre sí (Nina, 1992). Para esta investigación se utilizó una versión reducida del inventario que contiene 16 adjetivos, distribuido en tres dimensiones: estilo positivo (6 reactivos), estilo negativo (5 reactivos) y estilo reservado (5 reactivos). Los adjetivos derivan de la siguiente premisa inicial: "Cuando platico con mi pareja, soy". Algunos ejemplos de los adjetivos son: (a) amable, (b) dominante, y (c) débil. Los participantes calificaban del 1 (nada) al 5 (muy) la frecuencia con la que experimentan el adjetivo en referencia a la plática con su pareja. Se evaluó la consistencia interna para cada una de las dimensiones: estilo positivo $(\alpha=0.85)$, estilo negativo $(\alpha=0.82)$ y estilo reservado $(\alpha=0.79)$. Se observó una buena consistencia interna, de acuerdo con la prueba psicométrica Alfa de Cronbach.

\section{Escala de estrés en la relación marital.}

Este es un instrumento desarrollado por Nina y Barreto (2018), con el propósito de conocer la percepción de los sujetos cuando enfrentan situaciones estresantes en su relación marital. Posee 21 reactivos divididos en dos dimensiones: afrontamiento diádico (14 reactivos) y estrés (7 reactivos). Algunos ejemplos de los reactivos son: (a) Cuando algo me incomoda, lo expreso; (b) Ante los problemas trato de buscar solución; (c) Cuando siento estrés me aíslo; y (d) Cuando siento estrés me culpo. Estos reactivos se midieron mediante una escala Likert del 1 (Totalmente en desacuerdo) al 5 (Totalmente de acuerdo). Ambas dimensiones del instrumento muestran buena consistencia interna (afrontamiento $\alpha=0.92$ y estrés $\alpha=0.79$ ).

\section{Procedimiento}

Al recibir la aprobación del Comité Institucional para la Protección de Seres Humanos en la Investigación (CIPSHI) de la Universidad de Puerto Rico, Recinto de Río Piedras (UPRRP), se realizaron reuniones en varios lugares públicos, como centros de trabajo, centros comunitarios y universidades, con el propósito de contactar posibles participantes.
A los interesados se les explicó el estudio y se les indicó que informaran sobre su disponibilidad para participar.

Al comunicar su interés, se cotejó si en efecto las personas cumplían con los requisitos de participación. Luego de ello, se les presentó el consentimiento informado y, al firmarlo, se les aplicó el instrumento, el cual tomó aproximadamente 20 minutos para contestarlo. Es importante resaltar que cada participante en el estudio era uno de los integrantes de la relación marital. Los datos se recopilaron presencialmente, es decir, al completarlo, las personas lo entregaban en un sobre sellado al equipo de investigación.

\section{Análisis de datos}

Para los análisis estadísticos descriptivos e inferenciales, se utilizó el programa IBM SPSS versión 25. En cuanto a las variables sociodemográficas, se realizaron estadísticas descriptivas, con el propósito de conocer ciertas características de los participantes (género, educación, entre otras). Para evaluar las variables estilo de comunicación, estrés y afrontamiento diádico, se usaron estadísticos inferenciales. Se trabajó principalmente con regresión múltiple, a través de la cual se pretendía conocer la contribución de los estilos de comunicación en el afrontamiento diádico y el estrés marital.

\section{Resultados}

Respecto a la información descriptiva que se desprendió de los datos analizados, se destacan las siguientes puntuaciones promedio registradas por la muestra, en relación con las variables de interés: (a) estilo de comunicación positivo $(M=4.23$, $D E=0.68)$, (b) estilo de comunicación negativo $(M=2.29, D E=0.88)$, (c) estilo de comunicación reservado $(M=2.11, D E=0.85)$, (d) afrontamiento diádico $(M=3.99, D E=0.77)$, y (e) estrés en relación marital $(M=3.17, D E=0.85)$. De estos resultados, se evidenció cómo los participantes utilizan una comunicación positiva con su cónyuge. Además, identificaron varias prácticas de afrontamiento que se presentaban en el cuestionario como propias en su vida conyugal.

En primer lugar, se realizó una regresión múltiple en la que se evaluó qué tanto predicen 
los estilos de comunicación (positivo, negativo y reservado) y los niveles de afrontamiento diádico. De este análisis se puede resaltar que las variables relacionadas a los estilos de comunicación positivo y reservado resultaron ser estadísticamente significativas $(p=0.000)$. Incluso fue posible confirmar que las variables derivadas del estilo de comunicación explican el $35.2 \%$ de la variable dependiente afrontamiento diádico. Siendo el estilo de comunicación positivo $(\beta=0.46)$ la variable que mejor explica la variable de afrontamiento diádico (tabla 2). Le siguió el estilo de comunicación reservado, el cual presentó una asociación negativa ( $\beta=-0.19)$. Por último, la variable asociada al estilo de comunicación negativo no fue estadísticamente significativa $(\beta=-0.07, p=0.448)$.

Tabla 2

Regresión con las variables estilos de comunicaciones y afrontamiento en la relación marital

\begin{tabular}{lcccc}
\hline & $B$ & $E S B$ & $\beta$ & $p$ \\
\hline Constante & 2.30 & 0.56 & & 0.000 \\
Positivo & $(1.19,3.42)$ & & & \\
& 0.52 & 0.10 & 0.46 & 0.000 \\
Reservado & $(0.32,0.71)$ & & & 0.029 \\
Negativo & -0.17 & 0.08 & -0.19 & \\
& $(-0.33,-0.02)$ & & & 0.448 \\
\hline
\end{tabular}

Nota. $R^{2}=35.2 \%$

Fuente: elaboración propia.

En segundo lugar, se evaluó qué tanto predicen los estilos de comunicación (positivo, negativo y reservado) el estrés en la relación marital (tabla 3). De este análisis, se observó que solo la variable asociada al estilo de comunicación negativa resultó ser estadísticamente significativa $(\beta=0.25, p=$ 0.019). Esta fue la única variable que probó explicar significativamente la variable dependiente de estrés. Las variables asociadas a los estilos de comunicación positivo $(\beta=-0.003)$ y reservado $(\beta=0.10)$ no fueron estadísticamente significativas dentro del análisis de regresión realizado $(p>0.05)$. Es decir, estas no representaron un cambio significativo en la variable dependiente de estrés en la relación marital. Ante este panorama, las variables independientes, las cuales derivan del estilo de comunicación, apenas explican el $6.9 \%$ de la variable dependiente estrés en la relación marital.

Tabla 3

Regresión con las variables estilos de comunicaciones y estrés en la relación marital

\begin{tabular}{lcccc}
\hline & $B$ & $E S B$ & $\beta$ & $p$ \\
\hline Constante & 2.43 & 0.75 & & 0.002 \\
& $(0.95,3.91)$ & & & \\
Positivo & -0.004 & 0.13 & $>-0.00$ & 0.978 \\
& $(-0.27,0.26)$ & & & \\
Reservado & 0.10 & 0.10 & 0.10 & 0.335 \\
& $(-0.10,0.30)$ & & & \\
Negativo & 0.24 & 0.10 & 0.25 & 0.019 \\
& $(0.04,0.44)$ & & & \\
\hline
\end{tabular}

Nota. $\mathrm{R} 2=6.9 \%$

Fuente: elaboración propia. 
Al reevaluar los resultados, fue evidente que cuando la variable dependiente es el afrontamiento diádico, el estilo de comunicación positivo y reservado se asocia con esta de manera positiva y negativa, respectivamente. Incluso, estas variables predicen significativamente el afrontamiento diádico. Por otro lado, se observó que el estilo de comunicación negativo no fue un predictor significativo en el contexto de la relación de pareja. En cambio, cuando se estableció el estrés en las relaciones de pareja como variable dependiente, el estilo de comunicación negativo fue un predictor significativo del mismo. Pero no se comprobó que el estilo positivo y reservado fueran predictores significativos.

\section{Discusión}

En este estudio se buscaba evaluar la posible relación de los estilos de comunicación en el estrés y el afrontamiento diádico. Con relación a la contribución de los estilos de comunicación en la relación de pareja (positivo, negativo o reservado), en el estrés y el afrontamiento diádico, fue posible comprobar el modelo de Bodenmann (1995; 2005), en cuanto a la importancia que tiene la comunicación en la relación de pareja. Esto reafirma que la pareja se adapta al proceso de comunicación según las situaciones de tensiones o conflictos en su diario vivir.

Se observó que el estilo positivo tiene una relación positiva y significativa con el afrontamiento diádico. Esto sugiere que las personas casadas, al afrontar situaciones problemáticas, podrían utilizar estilos positivos de comunicación para lidiar con situaciones de la díada, lo que contribuye al desarrollo de estrategias positivas de afrontamiento (Ledermann et al., 2010). Este hallazgo sustenta el efecto que puede tener el estilo positivo en el bienestar psicológico de la pareja, al afrontar las situaciones conflictivas (Falconier y Kuhn, 2019). Estos resultados denotan una fortaleza en los participantes que utilizan estilos de comunicación positivos, ya que al emplearlos se puede incrementar la cohesión y los lazos emocionales sobre los que se sostiene la díada (Cutrona et al., 2018). Además, demuestran que este tipo de personas tienen una postura saludable cuando tienen que lidiar con los conflictos, por lo que desarrollan estrategias para poder manejarlos y mantener la relación estable.
Estos resultados son similares a los hallazgos de otras investigaciones que trabajan el tema, las cuales reconocen que la comunicación puede contribuir al afrontamiento de las parejas (Bodenmann et al., 2014; Hilpert et al., 2016; Nina, 2016; Randall y Bodenmann, 2009). Asimismo, explican por qué las parejas al utilizar un estilo de comunicación positivo pueden tener más posibilidades de afrontar de manera saludable sus problemas (Falconier et al., 2016; Nina, 1991; Sánchez y Watty, 2017; Waldherr y Muck, 2011), lo cual influye en un adecuado funcionamiento de la relación.

Por otra parte, el estilo reservado se relaciona de forma negativa con el afrontamiento diádico. Es decir, al afrontar situaciones conflictivas, no es característico utilizar un estilo de comunicación reservado. Emplear un estilo no expresivo es una barrera en el proceso de comunicación (SánchezAragón y Díaz-Loving, 2003), de manera que el rol de la persona pasa a ser pasivo cuando busca estrategias de afrontamiento que puedan mantener la estabilidad y la calidad de la relación. De hecho, los datos mostraron que no hay relación entre el estilo negativo y el afrontamiento diádico. En general, este análisis demuestra que los participantes del estudio tienen la capacidad de adaptarse a circunstancias problemáticas y superarlas por el bienestar psicológico de la pareja (Ledermann et al., 2010).

En el segundo análisis, en el que se sitúa el estrés en la pareja como variable dependiente, fue posible confirmar una relación positiva y significativa entre el estilo de comunicación negativo y el estrés diádico, lo que valida que las experiencias negativas que vive una persona casada pueden afectar el mantenimiento, la intimidad y la estabilidad de la relación marital (Karney y Bradbury, 1995). Estos resultados sustentan el hecho de que el estrés en parejas puertorriqueñas responde a aspectos que atañen a su relación y no a factores externos (Ledermann et al., 2010). Con esto se puede suponer que un estilo de comunicación negativo podría producir situaciones estresantes en la relación de pareja, generando frustración en sus integrantes, lo cual impacta en su salud emocional y física, así como en la satisfacción marital (Sánchez y Watty, 2017).

Los hallazgos del estudio permitieron identificar algunas identidades propias de la cultura de la pareja puertorriqueña. Es decir, una persona 
en una relación de pareja utiliza un estilo positivo en el afrontamiento diádico, pero también usa estilos negativos de comunicación cuando enfrenta estrés. Esto contradice lo que expone la teoría, en cuanto a que se supone que la pareja lidie con estilos positivos de comunicación tanto al tener estrés como al afrontarlos (Bodenmann, 2005; Falconier y Kuhn, 2019); lo que hace suponer que se está afectando la evolución y la continuidad del matrimonio. Por ello cabe preguntarse a qué responde que las personas ante el estrés no asuman un estilo positivo de comunicación. Con respecto a lo anterior, es fundamental reconocer la importancia del contenido de la comunicación frente al estilo, el estrés y el afrontamiento diádico (Bodenmann, 2005; Cutrona et al., 2018; Nina, 2016; SánchezAragón y Díaz-Loving, 2003; Sánchez y Watty, 2017).

Por otra parte, los resultados mostraron cómo la cultura tiene un efecto al afrontar el estrés, lo que sugiere que tanto la conducta individual o colectiva y la familia (extendida/nuclear) como los roles de género pueden estar influyendo en la construcción de la comunicación en la relación de pareja (Falconier et al., 2016).

Los resultados también evidenciaron que, al lidiar con el estrés y el afrontamiento, es más significativa la forma de expresarse de una persona en la relación de pareja que el contenido de lo que se habla (Kelley, 2012). Esta es una aportación al estudio de la pareja puertorriqueña, sobre todo a lo que atañe al tema de la comunicación y el afrontamiento diádico.

En síntesis, este estudio permitió conocer mejor el comportamiento de estas variables, demostrando cómo son las personas en sus relaciones de pareja al comunicarse y las implicaciones en el bienestar psicológico de la pareja. No obstante, esta investigación cuenta con varias limitaciones. Por un lado, el estudio adolece de la percepción de la pareja como unidad de análisis, por lo que para futuros estudios se propone considerar la pareja para tener una mirada más amplia del comportamiento de las variables de estudio. Otra limitación es que fue un estudio transversal y los datos fueron obtenidos en un solo momento y en el tiempo de la persona. Debido a ello, se sugiere explorar estas variables de forma longitudinal para poder ver los cambios en las diversas etapas de vida de la pareja. Asimismo, se recomienda en futuros estudios utilizar los diarios narrativos para examinar el comportamiento de esas variables en un periodo determinado.

Por otro lado, aunque no se abordó el contenido de la comunicación cuando se utilizan los estilos positivos para afrontar situaciones problemáticas, se sugiere continuar indagando tanto sobre la relación del contenido de la información y el estilo positivo como sobre la asociación entre las variables estudiadas, debido a que poco se conoce acerca del efecto del estilo de comunicación negativo en la relación marital. Además, la información que se brinda al utilizar estilos negativos ante el estrés diádico, puede servir para formular la pregunta de uno de los estudios futuros para darle continuidad al tema.

En cuanto a que es más significativa la forma de expresarse que el contenido cuando se lidia con el estrés y el afrontamiento, se sugiere desarrollar estudios futuros que aborden el tema del contenido de la comunicación, el estrés y el estilo de comunicación.

Por último, contar con un instrumento sobre estas variables abre las puertas a diversidad de líneas de investigación. De ahí que sea pertinente estudiar su relación con otros elementos, como la etapa de vida de la pareja, el tiempo de casados, la edad de los integrantes y la situación estresante que estén experimentando, además de otros factores asociados con el empleo, los problemas de salud y la relación con los hijos, entre otros.

Sin embargo, con estos resultados se espera desarrollar intervenciones psicoeducativas dirigidas a las parejas, con el propósito de enseñarles a utilizar destrezas adecuadas de comunicación (Lavner y Bradbury, 2017). Si bien se reconoce que el conflicto es parte del diario vivir, el mantenimiento de la relación depende del manejo adecuado de los conflictos (Bradbury y Karney, 2014), por ello es importante identificar las destrezas comunicativas positivas para hacer uso de estas. 


\section{Referencias}

Bodenmann, G. (1995). A Systemic-Transactional View of Stress and Coping in Couples. Swiss Journal of Psychology, 54, 34-49.

Bodenmann, G. (2005). Dyadic Coping and its Significance for Marital Functioning. In T. Revenson, K. Kayser, \& G. Bodenmann (Eds.), Couples Coping with Stress: Emerging Perspectives on Dyadic Coping (pp. 33-50). Washington, DC: American Psychological Association.

Bodenmann, G., Hilpert, P., Nussbeck, F. W., \& Bradbury, T. N. (2014). Enhancement of Couples Communication and Dyadic Coping by a Self-Directed Approach: A Randomized Controlled Trial. Journal of Consulting and Clinical Psychology, 82(4), 580591 doi:10.1037/a0036356

Bodenmann, G., Pihet, S., \& Kayser, K. (2006). The Relationship between Dyadic Coping and Marital Quality: A 2-Year Longitudinal Study. Journal of Family Psychology, 20(3), 485-493. doi:10.1037/0893-3200.20.3.485

Bradbury, T., \& Karney, B. (2014). Intimate Relationships. New York: Norton.

Cutrona, C. E., Bodenmann, G., Randall, A., Clavel, F., \& Johnson, M. (2018). Stress, Dyadic Coping and Social Support: Moving toward integration. In A. Vangelisti, \& D. Perlman (Eds.), The Cambridge Handbook of Personal Relationships (pp. 341-352). Cambridge: University Press.

Duck, S., \& McMahan, D. (2010). Communication in Everyday Life. Thousand Oaks: Sage Publication.

Falconier, M. K., \& Kuhn, R. (2019). Dyadic Coping in Couples: A Conceptual Integration and a Review of the Empirical Literature. Frontiers in Psychology, 10, 571. doi:10.3389/ fpsyg.2019.00571

Falconier, M. K., Randall, A. K., \& Bodenmann, G. (2016). Couples Coping with Stress: A CrossCultural Perspective. New York: Routledge. doi:10.4324/9781315644394

Finkel, E. J., Simpson, J. A., \& Eastwick, P. E. (2017). The Psychology of Close Relationships: Fourteen Core Principles. Annual Review of Psychology, 68, 383-411. doi:10.1146/ annurev-psych-010416-044038

Hilpert, P., Randall, A. K., Sorokowski, P., Atkins, D. C., Sorokowska, A., Ahmadi, K.,... Yoo, G. (2016). The Associations of Dyadic Coping and Relationship Satisfaction Vary between and within Nations: A 35-Nation Study. Frontiers Psychology, 7(1106), 1-16. doi:10.3389/ fpsyg.2016.01106

Karney, B., \& Bradbury, T. (1995). The Longitudinal Course of Marital Quality and Stability: A Review of Theory, Method, and Research. Psychological Bulletin, 118, 3-34.

Kelley, D. (2012). Marital Communication. Cambridge: Polity Press.

Lavner, J., \& Bradbury, T. (2017). Protecting Relationships from Stress. Current Opinion in Psychology, 13, 11-14. doi:10.1016/j. copsyc.2016.03.003

Ledermann, T., Bodenmann, G., Rudaz, M., \& Bradbury, T. (2010). Stress, Communication, and Marital Quality in Couples. Family Relations, 59, 195-206. doi: 10.1111/j.17413729.2010. 00595.x

Leuchtmann, L., Zemp, M., Milek, A., Nussbeck, F. W., Brandstaetter, V., \& Bodenmann, G. (2018). Role of Clarity of Other's Feelings for Dyadic Coping. Personal Relationships, 25(1), 38-49. doi:10.1111/pere.12226

Nina, R. (1991). Comunicación marital y estilos de comunicación: construcción y validación (Tesis de doctorado no publicada). Universidad Autónoma Nacional de México, México.

Nina, R. (1992). Inventario de estilo de comunicación marital: ECOM, su desarrollo y análisis psicométrico. Revista Puertorriqueña de Psicología, 8, 139-153.

Nina, R. (2016). Estrés y el afrontamiento diádico en la relación marital. Revista Griot, 9(1), 48-56.

Nina, R. y Barreto, E. (2018). Comunicación marital y estilo de comunicación positiva en afrontamiento diádico. Revista de Psicología Social y Personalidad, 34(2), 1-18.

Norton, R. (1978). Foundation of a Communicator Style Construct. Human Communication Research, 4, 99-112. 
Norton, R. (1983). Communicator Style: Theory, Applications and Measures. Beverly Hills, California: Sage Publication.

Orengo-Aguayo, R. (2015). Mexican American and Other Hispanic Couples' Relationship Dynamic: A Review to Inform Intervention Aimed at Promoting Healthy Relationships. Marriage \& Family Relations, 51(7), 633-667. doi/10.1080/01494929.2015.1068253

Randall, A. K., \& Bodenmann, G. (2009). The Role of Stress on Close Relationships and Marital Satisfaction. Clinical Psychology Review, 29(2), 105-115. doi:10.1016/j.cpr.2008.10.004

Razak, S., Hoesni, S, Zaharia, E., \& Ismail, R. (2015). The Role of Dyadic Coping to Marital Quality. Social and Behavioral Sciences, 211, 911-916.

Sánchez-Aragón, R. y Díaz-Loving, R. (2003). Patrones y estilos de comunicación de la pareja: Diseño de un inventario. Anales de Psicología, 19(2), 257-277.

Sánchez, C., \& Watty, A. (2017). Profiles Using Indicators of Marital Communication, Communication Styles, and Marital Satisfaction in Mexican Couples, Journal of Sex \& Marital Therapy, 43(4), 361-376. doi:10.1080/009262 3X.2016.1168332
Sillars, A., \& Vangelisti, A. (2018). Communication: Basic Properties and their Relevance to Relationship Research. In A. Vangelisti, \& D. Perlman (Eds.), The Cambridge Handbook of Personal Relationships (pp. 243-255). Cambridge: Cambridge University Press.

Uribe, I., García, M. y Ramírez, L. (2011). Relación entre estilos de comunicación y satisfacción sexual en estudiantes universitarios. Interamerican Journal of Psychology, 45(2), 157-167.

Waldherr, A., \& Muck, P. (2011). Towards an Integrative Approach to Communication Styles: The Interpersonal Circumplex and the Five-Factor Theory of Personality as Frames of Reference. Communications, 36(1), 1-27. doi:10.1515/comm.2011.001

Wijnberg-Williams, B., Van de Wiel, H. B. M., Kamps, W., \& Hoekstra-Weebers, J. (2015). Effects of Communication Styles on Marital Satisfaction and Distress of Parents of Pediatric Cancer Patients: A Prospective Longitudinal Study. Psycho-oncology, 24(1), 106-112. doi:10.1002/pon.3617

Para citar este artículo / To cite this article / Para citar este artigo: Nina-Estrella, R. y Marrero-Centeno, J. (2020). Estilos de comunicación como predictores del estrés y el afrontamiento diádico en matrimonios puertorriqueños. Pensamiento Psicológico, 18(1), 31-41. doi:10.11144/Javerianacali.PPSI18-1.ecpe 